University of Wollongong

Research Online

Faculty of Social Sciences - Papers (Archive) Faculty of Arts, Social Sciences \& Humanities

2013

CNV resolution does not cause NoGo anteriorisation of the P3: A failure to replicate Simson et al.

Janette Smith

University of New South Wales

Robert Barry

University of Wollongong, rbarry@uow.edu.au

Genevieve Steiner

University of Wollongong, gsteiner@uow.edu.au

Follow this and additional works at: https://ro.uow.edu.au/sspapers

Part of the Education Commons, and the Social and Behavioral Sciences Commons

Research Online is the open access institutional repository for the University of Wollongong. For further information contact the UOW Library: research-pubs@uow.edu.au 


\title{
CNV resolution does not cause NoGo anteriorisation of the P3: A failure to replicate Simson et al.
}

\begin{abstract}
For 35 years, some researchers have argued that CNV resolution may affect or even produce the increased P3 for NoGo compared to Go trials, and thus that no 'inhibitory' NoGo P3 exists. This is based on the work of Simson et al. (1977b), the scalp topography of potentials in auditory and visual Go/NoGo tasks. Electroencephalography and Clinical Neurophysiology, 43, 864-875, which compared Go and NoGo topography after CNV was subtracted from NoGo trials only. Specifically, the NoGo P3 topography showed the distinctive frontocentral maximum, which is often linked to motor inhibition, when referenced to a pre-target baseline. This NoGo topography changed to a more parietal maximum, similar to that on Go trials, when referenced to a pre-cue baseline. Many researchers have cited this study, while failing to use the delayed response design on which Simson et al. based their argument. We attempted to replicate Simson et al.'s experiment with delayed responses and also with immediate responses, as are more often used. As expected, the amplitudes of CNV and P3 to both Go and NoGo trials were increased when immediate compared to delayed responses were required, but we failed to replicate the topographic shift of NoGo P3 with different baselines for both delayed and immediate responses. That is, subtraction of the CNV from NoGo P3 did not change the distinctive frontocentral topography of this component. The results suggest that CNV may affect the amplitude and measurement of the NoGo P3, but that NoGo P3 anteriorisation is not caused by CNV resolution.
\end{abstract}

\section{Keywords}

replicate, simson, et, al, does, cnv, not, resolution, cause, nogo, anteriorisation, p3, failure

\section{Disciplines}

Education | Social and Behavioral Sciences

\section{Publication Details}

Smith, J. L., Barry, R. J. \& Steiner, G. Z. (2013). CNV resolution does not cause NoGo anteriorisation of the P3: A failure to replicate Simson et al.. International Journal of Psychophysiology, 89 (3), 349-357. 
CNV resolution does not cause NoGo anteriorisation of the P3: A failure to replicate Simson et al.

Janette L. Smith ${ }^{a}$, Robert J. Barry ${ }^{b}$, Genevieve Z. Steiner ${ }^{b}$

a. National Drug and Alcohol Research Centre, University of New South Wales, Sydney NSW 2052, Australia

b. Centre for Psychophysics, Psychophysiology, and Psychopharmacology; Brain \& Behaviour Research Institute; and School of Psychology, University of Wollongong, Wollongong NSW 2522, Australia

Corresponding author's address:

Janette L. Smith

National Drug and Alcohol Research Centre

University of New South Wales

Sydney NSW 2052 AUSTRALIA

Email: janette.smith@unsw.edu.au

Tel: (+612) 93850274

Fax: (+612) 93850222 


\section{Abstract}

For 35 years, some researchers have argued that CNV resolution may affect or even produce the increased P3 for NoGo compared to Go trials, and thus that no 'inhibitory' NoGo P3 exists. This is based on the work of Simson et al. (1977b, The scalp topography of potentials in auditory and visual go/nogo tasks. Electroencephalography and Clinical Neurophysiology, 43, 864-875), which compared Go and NoGo topography after CNV was subtracted from NoGo trials only. Specifically, the NoGo P3 topography showed the distinctive frontocentral maximum, which is often linked to motor inhibition, when referenced to a pre-target baseline. This NoGo topography changed to a more parietal maximum, similar to that on Go trials, when referenced to a pre-cue baseline. Many researchers have cited this study, while failing to use the delayed response design on which Simson et al. based their argument. We attempted to replicate Simson et al.'s experiment with delayed responses and also with immediate responses, as are more often used. As expected, the amplitudes of CNV and P3 to both Go and NoGo trials were increased when immediate compared to delayed responses were required, but we failed to replicate the topographic shift of NoGo P3 with different baselines for both delayed and immediate responses. That is, subtraction of the CNV from NoGo P3 did not change the distinctive frontocentral topography of this component. The results suggest that CNV may affect the amplitude and measurement of the NoGo P3, but that NoGo P3 anteriorisation is not caused by CNV resolution.

Keywords: Go/NoGo; inhibition; cue; fixed interval; component overlap; CNV. 


\section{Introduction}

Inhibitory processes can be examined in the Go/NoGo task, in which subjects must respond, usually with a button press, to one stimulus type (Go, usually at least $50 \%$ of trials, to ensure the Go response is prepotent), but withhold that response to stimuli of another type (NoGo, usually $50 \%$ or less trials, to ensure inhibition is rare and therefore more difficult). Eventrelated potentials (ERPs) show robust differences to these two stimulus types: on NoGo compared to Go trials, both N2 and P3 amplitudes are larger, and the P3 shows a more anterior topography (e.g., Bekker et al., 2004; Bruin \& Wijers, 2002; Kok, 1986; Lavric et al., 2004; Nieuwenhuis et al., 2003; Smith et al., 2006). The NoGo N2 is thought to be generated in the anterior cingulate cortex (e.g., Bekker et al., 2005) and to represent conflict between the competing Go and NoGo responses (e.g., Nieuwenhuis et al., 2003), while the NoGo P3 is thought to represent the action of the inhibitory process itself, and is associated with deactivation of motor areas used to make the Go response (Smith et al., in press).

The use of a cue to signal the identity and/or timing of an upcoming Go/NoGo stimulus allows simultaneous examination of preparatory processes, and increases the inhibitory requirements of the task, since a highly prepared response is harder to inhibit. The N2 and P3 effects are observed whether or not a cued task is used (e.g., uncued: Mathalon et al., 2003; Nieuwenhuis et al., 2003; Pfefferbaum \& Ford, 1988; cued: Jodo \& Kayama, 1992; Nativ et al., 1992; Roberts et al., 1994). Thus, the cue and the accompanying response preparation are not essential to understanding the N2 and P3 in the Go/NoGo task, at least where the cue is a predictor of target timing only, and not of target identity. However, in other inhibitory tasks with a cue-target trial structure, the processing of cue identity is integral to the interpretation of results in that task. For example, in the continuous 
performance task, as used by Roberts et al. (1994), subjects are required to respond to the target letter $(X)$ only if it is preceded by a particular cue letter $(A)$. The sequence $A-X$ is considered a response preparation-execution (Go) sequence, while the sequence $A-n o t-X$ is considered a response preparation-inhibition (NoGo) sequence. Thus, the letter A triggers preparatory processes and is important in successfully completing the task. In the Posner task (Posner et al., 1978), a cue stimulus informs the participant validly or invalidly of the location of the upcoming target. Thus the cue triggers a shift of spatial attention, which results in a reaction time (RT) benefit to validly cued targets, and a cost to invalidly cued targets, relative to when no cue or a neutral cue is presented. Some researchers have argued that response preparation on the cued side must be inhibited in the case of invalid cues, in order to give the correct response, thus, it is considered by some to be an inhibitory task (e.g., Gehring et al., 1992). The contingent negative variation (CNV; Walter et al., 1964) can be observed reliably in almost any task with a fixed cue-target interval. It is a slow negative shift arising between the cue and target, peaking at or just before target onset, and usually frontocentrally maximal ${ }^{1}$. Thus, the CNV is considered to be the electrophysiological marker of stimulus anticipation and response preparation processes.

\footnotetext{
${ }^{1}$ At least three separate waves contribute to the CNV: an early orienting response to the cue stimulus, often frontally negative and parietally positive (e.g., Connor and Lang, 1969; Weerts and Lang, 1973); the stimulus preceding negativity (SPN), a negative-going parietally maximal wave occurring when the participant expects a salient stimulus and most purely observed in the absence of a motor response to the stimulus (e.g., Damen and Brunia, 1985; Van Boxtel and Bocker, 2004); and the readiness potential (RP), a negative-going centrally and contralaterally maximal wave time-locked to the subject's response (Kornhuber and Deecke, 1964, 1965). The early and late waves can be separated by the use of a long cue-target interval or by use of principal components analysis (PCA). However, reliable separation and/or pure observation of the SPN and RP involves the use of vastly different experimental paradigms. In cue-target-response paradigms, stimulus anticipation and response preparation processes can never be observed alone, since the notion of preparing a response to some stimulus necessarily entails some expectancy for the stimulus. Similarly, in studies of the SPN, the expected stimulus often provides feedback about prior task performance and/or instructions about future tasks, and therefore it can be argued that SPN does have some aspect of preparation for future responses. Thus, the processes are not easily separable, and rather should be considered as "extremes on a perception-action continuum" (Van Boxtel and Bocker, 2004, p. 61).
} 
However, the cued paradigm causes problems with interpretation of post-target potentials, because the decay and termination of the negative shift of the CNV ("CNV resolution") is not necessarily instantaneous with the presentation of the target. Rather, CNV resolution may overlap with post-target potentials, thus possibly changing the observed amplitudes and topographies of ERP components occurring in that time range. Historical views of this problem have concentrated on the overlap of CNV with the P3 peak. It has been argued previously that the $\mathrm{P} 3$ was nothing more than the positive-going resolution of the CNV, not a component in itself (e.g., Donchin \& Smith, 1970; Wilkinson \& Ashby, 1974; Wilkinson \& Spence, 1973). This may be due partly to the fact that the P3 and CNV were first described at around the same time (Sutton et al., 1965; Walter et al., 1964) in similar tasks (i.e., cued designs with some degree of target stimulus uncertainty and relevance to the subjects' task). Indeed, the original paper by Walter et al. (1964) shows a P3-like overshoot of baseline after the target stimulus. The similarity in tasks eliciting CNV and P3 led other researchers to suggest that $\mathrm{CNV}$ and $\mathrm{P} 3$ were separable waves, but activated by a common mechanism. That is, the mechanism that increases CNV before task-relevant, compared to irrelevant stimuli, is the same mechanism that increases post-target potentials to taskrelevant stimuli (Näätänen, 1970). A flurry of work in the 1970s led to acceptance of the proposition that $\mathrm{CNV}$ and $\mathrm{P} 3$ are separate components, but that the positivity associated with CNV resolution may be added to the P3 positivity and hence influence the

\footnotetext{
The relative contribution of the SPN and RP to the 'late CNV' depends on the task requirements: in tasks which are primarily motoric, it is argued that the late CNV consists principally of response preparation processes, while in sensory tasks or in those which use meaningful or interesting stimuli requiring no response, the late CNV consists mostly of stimulus anticipation processes. We interpret the late CNV in this study as principally motoric; however, we do not believe this interpretation, or the possibility of separating SPN and RP, is important for the research question in this study.
} 
measurement of that component (see Donald \& Goff, 1971, 1973; Hillyard et al., 1976;

Karlin, 1970; Kok, 1988; Smith et al., 2007; Tueting \& Sutton, 1973; but especially Donchin et al., 1975; Verleger et al., in press). It should be noted here, as Oddy et al. (2005) have pointed out, that work has focused on the P3 despite the possibility that all post-target potentials are affected; for example, a possible relationship between the CNV and the N1 has been discussed by Jarvilehto and Fruhstorfer (1970), and Näätänen (1970) has argued that P2 amplitude is related to arousal in the prestimulus period, rather than any poststimulus processing.

After it was accepted that the CNV and P3 were separate processes, researchers often used a pre-cue baseline to study CNV and other post-cue processes, and a pre-target baseline to study post-target potentials, as if the electrical potentials occurring after the target were completely independent of those occurring before the target. This approach allows comparison with non-cued versions of tasks, which routinely have a pre-target baseline period, but ignores the contribution of CNV resolution, partly due to the lack of a method to remove it.

In the Go/NoGo task in particular, it has been an important problem, and Simson et al. (1977b) were the first to attempt a solution. Participants performed a cued task with equiprobable Go/NoGo stimuli, and were instructed to delay their response to the Go stimuli by about 1 second. The CNV developed over the cue-target interval as participants prepared a response, and showed the typical central maximum. Simson et al. argued that, on NoGo trials, CNV resolved almost immediately, since the subjects were required to do nothing further for the trial, but on Go trials, subjects maintained their state of readiness 
until it was appropriate to execute the response, thus delaying CNV resolution. After the target, the usual frontocentral increase in P3 for NoGo stimuli was observed (with a pretarget baseline; compare solid red (NoGo) and green (Go) lines in the left half of Figure 1). That is, a parietocentral maximum was observed for Go P3, while a central maximum was observed for NoGo P3. Simson et al. noted the similarity in central topographies for the CNV (blue lines in Figure 1) and NoGo P3, and argued that the immediate resolution of CNV on NoGo trials was the cause of the observed NoGo P3 central topography. When the NoGo P3 was referred to a pre-cue baseline (so as to minimise the influence of CNV development and resolution; dashed red lines in Figure 1), the Go/NoGo differences were no longer apparent. That is, when the CNV topography was removed in this fashion, the NoGo P3 amplitude was reduced and its topography was changed to a parietal maximum, similar to the Go P3 (see Figure 1). Therefore, Simson et al. suggested that the NoGo P3 effect was solely due to CNV resolution differences, not to any inhibitory process occurring on NoGo trials.

The method of using different baselines has been popular. Indeed, before Simson et al. (1977b) published their results, Donchin et al. (1975) measured both N1 and P3 relative to a pre-cue and a pre-target baseline. Other researchers continue to refer to Simson et al.'s method of using different baselines for Go and NoGo P3 (e.g., Roberts et al., 1994; Verleger et al., 2006), with results both similar to, and different from, those of Simson et al. (see Figure 1). For example, Verleger et al. reported effects similar to Simson et al., yet Roberts et al. reported that, after subtraction of the CNV from NoGo P3 amplitude, the topography was not similar to the centroparietally maximal Go P3, instead displaying a frontal maximum and retaining the frontal NoGo > Go effect. Still other studies do not perform the subtraction method, yet refer to differential CNV resolution as a possible explanation of 
their results, in contrast to an inhibitory explanation (e.g., Eimer, 1993; Jodo \& Inoue, 1990; Kok, 1986, 1988). However, none of those studies used a delayed response paradigm, which was the reason for the Go/NoGo differences in CNV resolution in Simson et al., and thus the crux of the argument for the use of differing baselines. Hence, due to the importance attributed to Simson et al.'s results despite no attempts to replicate them, in the current study we seek to discover whether we can reproduce the results of Simson et al. using delayed responses, and whether these effects are altered when an immediate response is used, as in more recent research. We compare our results to previously published work from Simson et al., Roberts et al., and Verleger et al., and report some notable similarities and differences between studies.

\section{Methods}

\subsection{Participants}

Participants were twenty adults ( 1 male, 1 left-handed) with a mean age of $22.9(S D=8.4)$ years who participated in the study for partial course credit. Self-reports indicated that none were on any psychotropic medication, had ever suffered a seizure, or had any hearing problems or uncorrected vision problems. All participants provided informed consent and the protocol was approved by the University of Wollongong's Human Research Ethics Committee before data collection began. 


\subsection{Stimuli and procedure}

Participants completed four versions in random order of a cued Go/NoGo task, using auditory or visual stimuli, and with immediate vs. delayed response required to Go stimuli. As far as possible, the task was designed to replicate Simson et al.'s (1976, 1977a, 1977b) methods, although in some instances information missing from those studies necessitated using modern standards (e.g., EOG correction, rejection of trials with large amplitude artefacts). On each trial a cue stimulus (S1) was followed 1000 ms later by the target stimulus (S2), which was either the same stimulus (in which case no response was required), or a different stimulus (in which case participants were required to make a button press response with the right index finger) with equal probability. The next cue stimulus appeared 7000 ms later. In each version of the task, 10 practice trials preceded 2 blocks of 40 trials.

In the auditory blocks, tones were $60 \mathrm{~ms}$ long (including $5 \mathrm{~ms}$ rise and decay times), presented binaurally through headphones at $60 \mathrm{~dB}$. The cue and NoGo stimuli were a 2000 $\mathrm{Hz}$ tone, while the Go stimulus was a $1000 \mathrm{~Hz}$ tone. The screen was black during the task, although participants were instructed to keep their eyes as still as possible throughout the task. In the visual blocks, the stimuli were black rectangles within a white circle $\left(3.4^{\circ}\right.$ visual angle) presented for $200 \mathrm{~ms}$ on a continuously black background. The cue and NoGo stimuli were vertically oriented rectangles, while the Go stimulus was a horizontally oriented rectangle. In the immediate response blocks, participants were instructed to 'press the button as quickly and accurately as possible', while in the delayed response blocks, participants were instructed to 'delay the response by about 1 second'. 


\subsection{Electrophysiological recording}

Continuous monopolar EEG was recorded from 30 scalp sites (Fp1, Fp2, F7, F3, Fz, F4, F8, FT7, FC3, FCz, FC4, FT8, T7, C3, Cz, C4, T8, TP7, CP3, CPz, CP4, TP8, P7, P3, Pz, P4, P8, O1, Oz, O2) using an elasticised cap with tin electrodes referenced to the left earlobe and grounded by an electrode located midway between FPz and Fz. Additional tin cup electrodes recorded activity from the right earlobe as well as vertical and horizontal EOG. Electrode impedances were below $5 \mathrm{k} \Omega$. Signals were amplified 500 times with a bandpass between 0.15 and 70 $\mathrm{Hz}$, and sampled at $1000 \mathrm{~Hz}$ using Neuroscan recording software.

\subsection{Data analysis}

The EEG was re-referenced to digitally-linked ears, filtered (lowpass at $30 \mathrm{~Hz}$, down 12 $\mathrm{dB} /$ octave, zero phase shift), and then corrected for eye movements using Neuroscan's inbuilt procedure (Semlitsch et al., 1986). Epochs began $1000 \mathrm{~ms}$ before the cue and ended $2000 \mathrm{~ms}$ after the target (4000 ms total), with a $100 \mathrm{~ms}$ pre-cue baseline. Trials with amplitudes exceeding $\pm 100 \mu \mathrm{V}$ in any scalp channel were rejected. Averages were created to Go and NoGo stimuli in each of the four versions of the task: Auditory Delayed, Visual Delayed, Auditory Immediate, and Visual Immediate. The CNV was measured as the mean amplitude in the last $100 \mathrm{~ms}$ before the target (when contributions from the early orienting wave are minimal, as shown by principal components analysis; e.g., Smith et al., 2006), while the P3 was measured as the most positive voltage $250-450$ ms after the target at $C z$, and then amplitude was measured at all other sites at the same latency (Picton et al., 2000). P3 measures were then also referred to a $100 \mathrm{~ms}$ pre-S2 baseline (equivalent to the late CNV mean amplitude measurement above), giving two P3 measures: 'P3-S1' describes P3 
amplitude relative to a pre-cue baseline, while 'P3-S2' describes P3 amplitude relative to a pre-target baseline.

\subsection{Statistical analyses}

Due to severe non-normality of error distributions, and the fact that few errors were made overall, no statistical analysis was performed on error data. For reaction time to Go stimuli, a $2 \times 2$ repeated measures ANOVA was performed with the factors Modality (Auditory, Visual) and Response (Delayed, Immediate). To examine the CNV elicited in each task, we ran a $2 \times 2 \times 2 \times 5$ repeated measures ANOVA with the factors Modality and Delay as above, as well as Type (before Go stimuli, before NoGo stimuli) and Site ( $F z, F C z, C z, C P z, P z)$. Orthogonal planned contrasts on the five equally spaced sites were used to examine simple (linear and quadratic) trends in the amplitudes over the scalp. A linear trend alone indicates a strong frontal or posterior distribution. A quadratic trend alone indicates a central maximum (or minimum). When both are significant, this indicates frontocentral or centroparietal distributions.

To determine whether we could replicate Simson et al.'s result, we examined Delayed and Immediate responses in separate analyses. In each analysis, we first examined the Go/NoGo topographic effects in each modality when both Go and NoGo P3 were referenced to a pretarget baseline, as is often done in cue-target paradigms. That is, we ran a Modality x Type (Go P3-S2, NoGo P3-S2) x Site ANOVA. Next, we followed Simson et al.'s method of referring only NoGo P3 to a pre-cue baseline to subtract the CNV topography. That is, we ran a Modality x Type (Go P3-S2, NoGo P3-S1) x Site ANOVA. Of course, direct comparison of 
NoGo P3-S1 with NoGo P3-S2 is not informative, as the difference between those conditions is simply the CNV topography. Replication of Simson et al.'s results would entail a significant Type $x$ Site interaction when both P3s were referenced to the pre-S2 baseline, and its absence when NoGo P3 was referenced to S1, denoting similar topography between types. As all contrasts were planned and there were no more of them than the degrees of freedom for effect, no Bonferroni-type adjustment to alpha was necessary (Tabachnick \& Fidell, 1996). Also, the single degree of freedom contrasts are not affected by violations of symmetry assumptions common in repeated measures analyses, and thus do not require Greenhouse-Geisser-type corrections. Where there were interactions of Modality, Type or Response with the Site factor, ERP data were submitted to vector scaling (McCarthy \& Wood, 1985) and only condition $x$ topography interactions that remained significant after this procedure are reported. All contrasts reported have $(1,19)$ degrees of freedom. We include the partial eta-squared $\left(\eta^{2}\right)$ statistic as an indicator of effect size throughout; it denotes the proportion of the effect + error variance attributable to the effect and can sum to more than 1.00 .

\section{Results}

\subsection{Behavioural performance}

Performance was generally good throughout the tasks. No participants made any commission errors to cue stimuli, and omission errors (failure to respond to Go stimuli, 0.4 $\%$ overall) and commission errors (responding to NoGo stimuli, $0.8 \%$ overall) were very infrequent in every condition, although commission errors were more likely for Immediate 
than Delayed responses (1.6\% vs. $0.1 \%$ ). Reaction time was significantly longer in the Delayed conditions ( $1011.7 \mathrm{~ms}, \mathrm{SD}=307.7 \mathrm{~ms}$ ) compared to the Immediate conditions $\left(349.2 \mathrm{~ms}, \mathrm{SD}=52.2 \mathrm{~ms} ; \mathrm{F}=100.047, \mathrm{p}<.001\right.$, partial $\left.\eta^{2}=.840\right)$, with no main effect or interaction for the visual vs. auditory modalities (700.0 ms (SD = $183.4 \mathrm{~ms}$ ) vs. $660.9 \mathrm{~ms}$ (SD $=181.0 \mathrm{~ms}$ ); main effect: $\mathrm{F}=1.186, p=.290$, partial $\eta^{2}=.059$; interaction $\mathrm{F}<1$ ). Thus, participants appear to have heeded task instructions well.

\subsection{Do we observe a similar CNV to Simson et al.?}

Figure 2 shows grand mean waveforms at midline sites in this study, while Figure 3 (blue lines) shows CNV amplitude, and Figure 4 shows CNV topography across the scalp.

Unsurprisingly, there were no main effects or interactions involving the Type factor, indicating that CNV was not significantly different before Go and NoGo stimuli (as would be expected from the cue not predicting target identity). Thus, Figures 3 and 4 show data averaged across Type. CNV showed a frontocentral maximum, with larger amplitudes anteriorly than posteriorly $\left(F=8.005, p=.011\right.$, partial $\left.\eta^{2}=.296\right)$, and much larger CNV amplitudes centrally $\left(F=95.746, p<.001\right.$, partial $\left.\eta^{2}=.834\right)$. There was a significant main effect of Delay, such that larger CNV amplitudes were observed in the Immediate compared to Delayed response condition $\left(F=15.076, p=.001\right.$, partial $\left.\eta^{2}=.442\right)$. There were significant Modality $x$ Site interactions, such that CNV was larger anteriorly $(F=21.048, p<.001$, partial $\left.\eta^{2}=.526\right)$ and larger centrally $\left(F=5.059, p=.037\right.$, partial $\left.\eta^{2}=.210\right)$ for Auditory than Visual stimuli. No other interactions were significant. 
Overall, it appears that similar CNV before delayed responses was elicited in both Simson et al. (1977b) and this study, although the sharply reduced amplitudes at CPz and Pz for auditory stimuli observed by Simson et al. were not replicated here. For immediate responses, we have again elicited similar topographic effects to Roberts et al. (1994) and Verleger et al. (2006), although amplitude is generally reduced.

\subsection{Are Simson et al.'s results replicable? The case of delayed responses}

Inspection of the left side of Figure 1 reveals that in Simson et al.'s (1977b) data, the NoGo P3 (solid red) was larger than Go P3 (solid green) at all sites when referred to a pre-S2 baseline, particularly at central sites, similar to the CNV topography, but that when NoGo P3 was referred to a pre-S1 baseline (dashed red), there was little difference in amplitude and topography to Go P3 (still referred to a pre-S2 baseline). That is, after subtraction of the CNV topography from NoGo P3, it displayed a Go-like centroparietal maximum topography.

Inspection of the left side of Figure 3 reveals that such an effect was not replicated in our data. We observed a typical P3 NoGo effect (see Figures 4 and 5), with larger amplitudes for NoGo than Go at frontocentral sites, and the reverse observed at posterior sites, for both auditory and visual stimuli. However, when NoGo P3 is referred to a pre-cue baseline, amplitudes are not similar to Go P3, and the topography retains its distinctive frontocentral maximum for both modalities. 
This was confirmed in separate Modality $x$ Type $x$ Site ANOVAs comparing Go P3-S2 with NoGo P3-S2 and with NoGo P3-S1. When both Go P3 and NoGo P3 were referred to a pre-S2 baseline, a significant centroparietal maximum was observed across types, with posterior > anterior $\left(F=27.421, p<.001\right.$, partial $\left.\eta^{2}=.591\right)$ and central $>$ anterior/posterior effects $(F=$ 126.700, $p<.001$, partial $\left.\eta^{2}=.870\right) . P 3$ tended to be larger in the visual than auditory blocks $\left(F=3.259, p=.087\right.$, partial $\left.\eta^{2}=.146\right)$, as well as showing a larger posterior $>$ anterior effect $\left(F=7.938, p=.011\right.$, partial $\left.\eta^{2}=.295\right)$. There was no significant main effect of Type $(F<1)$, but there were highly significant Type $x$ Site interactions, confirming the more anterior focus of the NoGo compared to Go P3. That is, the NoGo > Go effect was larger anteriorly than posteriorly $\left(F=16.672, p=.001\right.$, partial $\left.\eta^{2}=.467\right)$, and larger centrally than anteriorly/posteriorly $\left(F=7.857, p=.011\right.$, partial $\left.\eta^{2}=.293\right)$.

When NoGo P3 was referred to a pre-S1 baseline (while Go P3 was still referred to a pre-S2 baseline, as per Simson et al., 1977b), the centroparietal maximum was retained, with a significant posterior $>$ anterior effect $\left(F=28.947, p<.001\right.$, partial $\left.\eta^{2}=.604\right)$, and a significant central $>$ anterior/posterior effect $\left(F=117.253, p<.001\right.$, partial $\left.\eta^{2}=.861\right)$. The visual $>$ auditory effect was now significant $\left(F=7.345, p=.014\right.$, partial $\left.\eta^{2}=.279\right)$, although the topographic effects were no longer significant $(F<2.647)$, nor were any other interactions involving modality. A Type main effect was significant $(F=13.973, p=.001$, partial $\left.\eta^{2}=.424\right)$, indicative of globally reduced NoGo P3 amplitude when referred to a precue baseline. However, most importantly, topographic interactions with Type remained significant: NoGo P3-S1 showed a frontocentral maximum, while Go P3-S2 showed a 
centroparietal maximum, reflected in a strong posterior > anterior gradient for Go stimuli, and the absence of this gradient for NoGo stimuli $\left(F=22.921, p<.001\right.$, partial $\left.\eta^{2}=.547\right)$.

\subsection{Are Simson et al.'s results replicable? The case of immediate responses}

In Simson et al.'s (1977b) study, participants were required to delay their response to Go stimuli by 'about 1 second', thus giving rise to the possibility of CNV resolution occurring immediately on NoGo trials, and being delayed for Go trials. Roberts et al. (1994) and Verleger et al. (2006) have followed Simson et al.'s method more recently, although their participants (and indeed, participants in most cued-Go/NoGo paradigms) responded immediately to Go stimuli. Some similarities and differences with respect to Simson et al.'s results are observed (compare right portion of Figure 1). Clearly, larger P3s and CNVs are elicited by immediate responses, as well as larger NoGo > Go effects at frontal sites. Verleger et al.'s results are similar to Simson et al.'s visual results, with reduced amplitudes and a centroparietal maximum for NoGo stimuli after CNV subtraction, however, for Roberts et al., amplitudes are larger frontally than parietally for NoGo stimuli, and a classic frontal NoGo > Go effect is maintained. Thus, we compare our results in the visual modality with theirs, as well as results in the auditory modality. In both modalities, again, the distinctly frontocentral maximum for NoGo stimuli is maintained after CNV subtraction, although a NoGo > Go effect at frontocentral sites is observed only for the visual modality.

This was confirmed statistically with ANOVAs as above (see Figure 3): When Go and NoGo P3 were both referred to a pre-target baseline, a centroparietal maximum was displayed 
(posterior $>$ anterior: $\mathrm{F}=26.805, \mathrm{p}<.001$, partial $\eta^{2}=.585$; central $>$ anterior/posterior: $\mathrm{F}=$ 129.864, $\mathrm{p}<.001$, partial $\eta^{2}=.872$ ). Larger P3s were observed in the visual than auditory modality $\left(F=15.229, p=.001\right.$, partial $\left.\eta^{2}=.445\right)$, with this effect tending to be larger for NoGo than Go stimuli $\left(F=3.467, p=.078\right.$, partial $\left.\eta^{2}=.154\right)$. There was no significant main effect of Type $\left(F=1.855, p=.189\right.$, partial $\left.\eta^{2}=.089\right)$, but there were significant topographic differences between Go and NoGo responses, indicative of the more frontocentral distribution for NoGo stimuli, reflected in a stronger posterior > anterior effect for Go than NoGo trials $\left(F=65.992, p<.001\right.$, partial $\left.\eta^{2}=.776\right)$, and a stronger central $>$ anterior/posterior effect for NoGo trials $\left(F=30.217, p<.001\right.$, partial $\left.\eta^{2}=.614\right)$.

When NoGo P3 was referred to a pre-cue baseline, the centroparietal maximum was maintained (posterior $>$ anterior: $\mathrm{F}=27.960, \mathrm{p}<.001$, partial $\eta^{2}=.595$; central $>$ anterior/posterior: $F=123.468, p<.001$, partial $\left.\eta^{2}=.867\right)$. The visual $>$ auditory effect was still significant $\left(F=18.944, p<.001\right.$, partial $\left.\eta^{2}=.499\right)$, with this effect now significantly larger for NoGo than Go stimuli $\left(F=4.801, p=.041\right.$, partial $\left.\eta^{2}=.202\right)$, although the topographic differences for visual and auditory stimuli were no longer significant. A Type main effect was now significant $\left(F=30.782, p<.001\right.$, partial $\left.\eta^{2}=.618\right)$, due to the decreased amplitude of NoGo P3 after CNV subtraction. However, the topographic differences for Go and NoGo stimuli were maintained, with a stronger posterior > anterior gradient for Go than NoGo trials $\left(F=70.943, p<.001\right.$, partial $\left.\eta^{2}=.789\right)$. 


\section{Discussion}

The overlap of CNV resolution with post-target potentials is a long-standing and important problem. It bears not only on cued Go/NoGo tasks as used here, but also on other cued tasks such as the Posner task and continuous performance task (A-X type), and indeed, on any task in which the CNV may be elicited - including any task design with a fixed interstimulus interval. There is currently no universally accepted method to deal with CNV resolution effects on Go/NoGo target potentials. Although PCA-based approaches are lately gaining ground (Oddy et al., 2005; Verleger et al., in press), they are yet to reach wide acceptance or implementation. Simson et al.'s (1977b) method of referring Go and NoGo P3 to different baselines, and the conclusion that the Go/NoGo P3 topographic effect may be solely due to differential CNV resolution on Go and NoGo trials, has been highly cited, receiving an average of 6 citations per year in the 35 years since its publication. Other researchers have also used the method (Roberts et al., 1994; Verleger et al., 2006), and have found some similar and some different results. However, the papers citing Simson et al. as well as those using their method of differing baselines have required immediate rather than delayed responses, although the use of delayed responses was the sole reason that temporally different CNV resolution might be a possibility for Go and NoGo trials in the Simson et al. study. This is because, under delayed response conditions, the participant must maintain their response readiness (and a high amplitude CNV) after a Go stimulus is presented, but has nothing further to do when a NoGo stimulus is presented, so CNV may resolve immediately. Under immediate response instructions, CNV may resolve quickly on both Go and NoGo trials. Thus, the current research addresses an important methodological question concerning the use of different baselines to 'remove' CNV resolution overlap from post-target potentials, and whether Simson et al.'s highly cited results were reproducible. 
We attempted to replicate the methodology of Simson et al. (1977b) as far as possible, with the addition of the immediate response condition. However, some information was missing from Simson et al. (1976, 1977a, 1977b), which necessitated the application of arbitrary decisions or using modern standards (e.g., Picton et al., 2000). For example, Simson et al. did not specify which hand or finger was used to respond; we chose to use the right index finger across all participants, since this is commonly used in many cued Go/NoGo paradigms. Although the supraorbital waveform (channel 0) in their Figure 2 (Simson et al., $1977 b)$ is relatively flat, suggesting eye movements have not contributed substantially to the observed scalp waveform averages, they do not detail how EOG or other artefacts were dealt with; thus, we have used modern regression-based EOG correction methods and standard scalp artefact rejection criteria. We do not consider that these points of difference between methods are responsible for the differences in results reported here and in Simson et al.

We also consider it unlikely that the difference in reference electrode between studies is responsible for the changes in topographic effects. Simson et al. (1977b) used a nose reference, Roberts et al. (1994) recorded using a Cz reference and later re-referenced to the earlobes, Verleger et al. (2006) used linked mastoids, and here we recorded using a left ear reference and later re-referenced offline to digitally-linked earlobes. However, the issue at stake in this study is the topographic similarities and differences in Go P3, NoGo P3, and the CNV. Even if the earlobes are active, and show stimulus-locked activity relative to the nose, re-referencing to linked earlobes will result in the same value being subtracted from all 
scalp sites, producing no change in scalp topography although a change in amplitude is possible. Also, since impedances were below $5 \mathrm{k} \Omega$ for all recorded sites, differential noise at the left and right earlobe should not have interfered with topography, particularly since our analyses focus on midline rather than hemispheric sites. Since we did not record the activity at the tip of the nose, we cannot say for certain that a difference in reference electrode is not the cause of the different effects for our study and for Simson et al. However, we think it unlikely that the difference in reference electrode location can account for the different results we observed.

In our study we examined CNV as the mean amplitude over the last $100 \mathrm{~ms}$ before the target, which differs from Simson et al.'s (1977b) peak amplitude measurement at $100 \mathrm{~ms}$ before the target, but is similar to many other studies using this type of task. We elicited a CNV in line with expectations from previous studies, being larger (and more strongly lateralised in Figure 4) for Immediate compared to Delayed responses (compare Simson et al.'s data with Roberts et al., 1994, and Verleger et al., 2006), and the CNV in the Delayed condition was similar in amplitude to Simson et al.'s CNV. Two points of difference arise from the CNV data of Simson et al.: First is that their Go waveforms (in their Figure 2) show a second negativity arising after post-target potentials, suggesting that CNV may resolve and then a second readiness potential develops before the delayed response. We saw no evidence of such a negative shift in our data, with stable amplitude maintained in the Delayed conditions at 500-1000ms post-target, when the response occurred (see Figure 5). Second, our CNV topography was relatively symmetrical about $\mathrm{Cz}$, with similar amplitudes at $\mathrm{FCz}$ and $\mathrm{CPz}$, and at $\mathrm{Fz}$ and Pz. In contrast, the CNV elicited in Simson et al. displayed a 
distinctly frontocentral topography for the auditory condition, with a sharp drop-off of CNV amplitude at $\mathrm{CPz}$ and $\mathrm{Pz}$. It is unlikely that this topography is a consequence of the nose reference used by Simson et al.; reduction of EEG amplitude can occur at sites close to the reference, yet this would rather produce small frontal, rather than small parietal amplitudes. This atypical topography, subtracted from the NoGo P3 topography only, may be responsible for the parietal maximum observed for NoGo P3-S1 in Simson et al., as discussed below.

Simson et al. (1977b) observed that the topography of the NoGo P3 (referenced to a pre-S2 baseline) and the CNV were similar (both being largest centrally), and proposed that the positive-going resolution of the CNV, which happens quickly on NoGo but not their delayed Go trials, might be responsible for the central maximum of the NoGo P3, compared to the parietally maximal Go P3. After subtracting the CNV, NoGo P3-S1 showed a parietal maximum, similar to the Go P3. Simson et al. concluded that the differential resolution of CNV on Go and NoGo trials was the cause of the frontocentrally maximal NoGo > Go P3 effect, and this has been highly cited in the literature as an alternative to the inhibitory explanation of this effect (e.g., Kok, 1988). In the first attempt to reproduce this effect with delayed responding in the 35 years since its publication, we did not replicate the parietal shift in topography for the NoGo P3-S1. In our study, a distinctly frontocentrally maximal topography was retained after CNV subtraction for both Auditory Delayed and Visual Delayed conditions. We consider that Simson et al.'s atypical CNV topography may be the reason for this effect. That is, an abnormally small CNV was subtracted from NoGo P3-S2 at $\mathrm{CPz}$ and $\mathrm{Pz}$, resulting in too much positivity being retained at those sites, and hence the 
NoGo P3-S1 showed a more parietally distributed topography than it should. With only two studies examining delayed responses, it is difficult to tell which reports the 'more correct' CNV topography; further research may assist in this area.

The notion of differential CNV resolution is theoretically plausible for delayed responses, but the rationale for differential resolution for immediate responses is less clear, yet most cued Go/NoGo studies require immediate responses. Therefore, we also examined the effect of CNV subtraction from NoGo P3 with Immediate responses. In contrast to Simson et al.'s (1977b) parietally maximal NoGo P3-S1, Roberts et al. (1994) reported a frontal maximum after CNV subtraction, and the classic frontal NoGo > Go P3 effect was retained. In Verleger et al. (2006), like Simson et al., the NoGo P3 shifted from a central maximum to a centroparietal maximum with CNV subtraction, but, unlike Simson et al., it still did not display a topography similar to Go P3. In our Immediate response data, as with the Delayed responses, the frontocentral maximum for NoGo P3 was retained after CNV subtraction, for both Auditory and Visual conditions.

The argument in this study rests on the notion that, in contrast to the pre-target baseline, the pre-cue baseline contains no contribution from CNV. This assumption would be negated by the presence of a CNV in the pre-cue period. It is possible, with the fixed 7000ms targetto-cue interval used in this study, that a CNV would arise in that interval; certainly, other researchers have observed CNV with such a long interstimulus interval (Connor \& Lang, 1969; Weerts \& Lang, 1973). However, for all conditions, we note the flatness of the pre-cue 
period $(-2000 \mathrm{~ms}$ to $-1000 \mathrm{~ms})$ in Figure 2 , indicating the absence of a pre-cue late CNV, as well as the absence of a frontal-negative, parietal-positive 'orienting' wave peaking around 2000 ms post-target. Thus, our assumption that the pre-cue baseline contains no contribution from CNV is upheld.

Simson et al.'s (1977b) conclusion that differential CNV resolution on NoGo compared to Go trials is the cause of the NoGo P3 topographic difference appears to be incorrect. The retention of the topographic effect after CNV subtraction suggests it is independent of CNV processes, and is likely due to other post-target processes. Given our previous work in the area (e.g., Smith et al., in press; Smith et al., 2008), we consider motor or cognitive inhibition to be highly probable candidates for such post-target processes producing the NoGo P3 effect, although consideration of these theories is outside the scope of this article. Although we argue that extension of the method to Immediate responses is unwarranted, we nonetheless wished to compare the results obtained with Immediate responses using the same subtraction method, and observed, again, that temporally different resolution of the CNV cannot account for the NoGo P3 topography. Our results suggest that CNV may affect the amplitude and measurement of the NoGo P3, but we argue that the NoGo P3 topography is not caused by CNV resolution, either for delayed or immediate responses. It follows that 'correction' of Go/NoGo P3 amplitudes by baseline subtraction has no established value and should not be implemented in future research. 


\section{Acknowledgements}

Acknowledgements: Thanks are due to Mr Tony Kemp who wrote the stimulus presentation program used in the study.

\section{Funding}

Funding: The study was supported by a UNSW Vice-Chancellor's Postdoctoral Fellowship to JS. The National Drug and Alcohol Research Centre at the University of NSW is supported by funding from the Australian Government under the Substance Misuse Prevention and Service Improvements Grants Fund.

\section{References}

Bekker, E. M., Kenemans, J. L., \& Verbaten, M. N. (2004). Electrophysiological correlates of attention, inhibition, sensitivity and bias in a continuous performance task. Clinical Neurophysiology, 115(9), 2001-2013.

Bekker, E. M., Kenemans, J. L., Verbaten, M. N. (2005) Source analysis of the N2 in a cued Go/NoGo task. Cognitive Brain Research 22:221-231.

Bruin, K. J., \& Wijers, A. A. (2002). Inhibition, response mode, and stimulus probability: a comparative event-related potential study. Clinical Neurophysiology, 113, 1172-1182. Connor, W. H., \& Lang, P. J. (1969). Cortical slow-wave and cardiac rate responses in stimulus orientation and reaction time conditions. Journal of Experimental Psychology, 82(2), 310-320.

Damen, E. J. P., \& Brunia, C. H. M. (1985). Slow brain potentials related to movement and visual feedback in a response timing task. Biological Psychology, 20, 195. 
Donald, M. W., \& Goff, W. R. (1971). Attention-related increases in cortical responsivity dissociated from the contingent negative variation. Science, 172, 1163-1166.

Donald, M. W., \& Goff, W. R. (1973). Contingent negative variation and sensory evoked responses: their interaction and relationship to auditory discrimination.

Electroencephalography and Clinical Neurophysiology, Supplement 33, 109-117.

Donchin, E., \& Smith, D. B. D. (1970). The contingent negative variation and the late positive wave of the average evoked potential. Electroencephalography and Clinical Neurophysiology, 29, 201-203.

Donchin, E., Tueting, P., Ritter, W., Kutas, M., \& Heffley, E. F. (1975). On the independence of the CNV and the P300 components of the human averaged evoked potential.

Electroencephalography and Clinical Neurophysiology, 38, 449-461.

Eimer, M. (1993). Effects of attention and stimulus probability on ERPs in a Go/Nogo task. Biological Psychiatry, 35, 123-138.

Gehring, W. J., Gratton, G., Coles, M. G. H., \& Donchin, E. (1992). Probability effects on stimulus evaluation and response processes. Journal of Experimental Psychology: Human Perception and Performance, 18(1), 198-216.

Hillyard, S. A., Courchesne, E., Krausz, H. I., \& Picton, T. W. (1976). Scalp topography of the P3 wave in different auditory decision tasks. In W. C. McCallum \& J. R. Knott (Eds.), The responsive brain (pp. 81-87). Bristol: John Wright and sons.

Jarvilehto, T., \& Fruhstorfer, H. (1970). Differentiation between slow cortical potentials associated with motor and mental acts in man. Experimental Brain Research, 11, 309-317. Jodo, E., \& Inoue, K. (1990). Effects of practice on the P300 in a Go/NoGo task. Electroencephalography and Clinical Neurophysiology, 76, 249-257. 
Jodo, E., \& Kayama, Y. (1992). Relation of a negative ERP component to response inhibition in a Go/No-go task. Electroencephalography and Clinical Neurophysiology, 82(6), 477-482. Karlin, L. (1970). Cognition, preparation, and sensory-evoked potentials. Psychological Bulletin, 73(2), 122-136.

Kok, A. (1986). Effects of degradation of visual stimuli on components of the event-related potential (ERP) in go/nogo reaction tasks. Biological Psychology, 23(1), 21-38.

Kok, A. (1988). Overlap between P300 and movement-related-potentials: A response to Verleger. Biological Psychology, 27, 51-58.

Kornhuber, H. H., \& Deecke, L. (1964). Hirnpotentialänderungen beim Menschen vor und nach Willkürbewegungen, dargestellt mit Magnetbandspeicherung und Rückwärtsanalyse. Pflügers Archiv, 281, 52.

Kornhuber, H. H., \& Deecke, L. (1965). Hirnpotentialänderungen bei Willkürbewegungen und passive bewegungen des menschen: Bereitschaftspotential une reafferente potentiale. Pflügers Archiv, 284, 1-17.

Lavric, A., Pizzagalli, D. A., \& Forstmeier, S. (2004). When 'go' and 'nogo' are equally frequent: ERP components and cortical tomography. European Journal of Neuroscience, 20, $2483-2488$.

Mathalon, D. H., Whitfield, S. L., \& Ford, J. M. (2003). Anatomy of an error: ERP and fMRI. Biological Psychology, 64(1-2), 119-141.

McCarthy, G., \& Wood, C. C. (1985). Scalp distributions of event-related potentials: An ambiguity associated with analysis of variance models. Electroencephalography and Clinical Neurophysiology: Evoked Potentials, 62(3), 203-208.

Näätänen, R. (1970). Evoked potential, EEG, and slow potential correlates of selective attention. Acta Psychologica, 33, 178-192. 
Nativ, A., Lazarus, J.-a. C., Nativ, J., \& Joseph, J. (1992). Potentials associated with the initiation and inhibition of visually triggered finger movement in humans: The "NoGo potential" in the Go/NoGo paradigm. International Journal of Neuroscience, 66, 107-118. Nieuwenhuis, S., Yeung, N., van den Wildenberg, W., \& Ridderinkhof, K. R. (2003). Electrophysiological correlates of anterior cingulate function in a go/no-go task: Effects of response conflict and trial type frequency. Cognitive, Affective and Behavioral Neuroscience, $3(1), 17-26$.

Oddy, B. W., Barry, R. J., Johnstone, S. J., \& Clarke, A. R. (2005). Removal of CNV effects from the N2 and P3 ERP components in a visual Go/NoGo task. Journal of Psychophysiology, 19(1), 24-34.

Pfefferbaum, A., \& Ford, J. M. (1988). ERPs to stimuli requiring response production and inhibition: effects of age, probability and visual noise. Electroencephalography and Clinical Neurophysiology, 71, 55-63.

Picton, T. W., Bentin, S., Berg, P., Donchin, E., Hillyard, S. A., Johnson, R., et al. (2000). Guidelines for using human event-related potentials to study cognition: Recording standards and publication criteria. Psychophysiology, 37, 127-152.

Posner, M. I., Nissen, M. J., \& Ogden, W. C. (1978). Attended and unattended processing modes: The role of set for spatial location. In H. L. Pick \& E. Saltzman (Eds.), Modes of perceiving and processing information (pp. 137-157). Hillsdale, New Jersey: Lawrence Erlbaum Associates.

Roberts, L. E., Rau, H., Lutzenberger, W., \& Birbaumer, N. (1994). Mapping P300 waves onto inhibition: Go/No-Go discrimination. Electroencephalography and Clinical Neurophysiology, $92,44-55$. 
Semlitsch, H. V., Anderer, P., Schuster, P., \& Presslich, O. (1986). A solution for reliable and valid reduction of ocular artifact, applied to the P300 ERP. Psychophysiology, 23(6), 695-703. Simson, R., Vaughan, H. G., \& Ritter, W. (1976). The scalp topography of potentials associated with missing visual or auditory stimuli. Electroencephalography and Clinical Neurophysiology, 40, 33-42.

Simson, R., Vaughan, H. G., \& Ritter, W. (1977a). The scalp topography of potentials in auditory and visual discrimination tasks. Electroencephalography and Clinical Neurophysiology, 42(4), 528-535.

Simson, R., Vaughan, H. G., \& Ritter, W. (1977b). The scalp topography of potentials in auditory and visual go/nogo tasks. Electroencephalography and Clinical Neurophysiology, 43, 864-875.

Smith, J. L., Jamadar, S., Provost, A. L., \& Michie, P. T. (in press). Motor and non-motor inhibition in the Go/NoGo task: An ERP and fMRI study. International Journal of Psychophysiology.

Smith, J. L., Johnstone, S. J., \& Barry, R. J. (2006). Effects of pre-stimulus processing on subsequent events in a warned Go/NoGo paradigm: Response preparation, execution and inhibition. International Journal of Psychophysiology, 61, 121-133.

Smith, J. L., Johnstone, S. J., \& Barry, R. J. (2007). Response priming in the Go/NoGo task: The N2 reflects neither inhibition nor conflict. Clinical Neurophysiology, 118, 343-355. Smith, J. L., Johnstone, S. J., \& Barry, R. J. (2008). Movement-related potentials in the Go/NoGo task: The P3 reflects both cognitive and motor inhibition. Clinical Neurophysiology, 119(3), 704-714.

Sutton, S., Braren, M., Zubin, J., \& John, E. R. (1965). Evoked-potential correlates of stimulus uncertainty. Science, 150, 1187-1188. 
Tabachnick, B. G., \& Fidell, L. S. (1996). Using multivariate statistics (Third ed.). New York: HarperCollins.

Tueting, P., \& Sutton, S. (1973). The relationship between prestimulus negative shifts and poststimulus components of the averaged evoked potential. In S. Kornblum (Ed.), Attention and Performance IV (pp. 185-207). New York: Academic press.

Van Boxtel, G. J. M., \& Böcker, K. B. E. (2004). Cortical measures of anticipation. Journal of Psychophysiology, 18, 61-76.

Verleger, R., Paehge, T., Kolev, V., Yordanova, J., \& Jaskowski, P. (2006). On the relation of movement-related potentials to the go/no-go effect on P3. Biological Psychology, 73(3), 298-313.

Verleger, R., Paulick, C., Möcks, J., Smith, J. L., \& Keller, K. (in press). Parafac and Go/No-Go: Disentangling CNV return from the P3 complex by Trilinear Component Analysis. International Journal of Psychophysiology.

Walter, W. G., Cooper, R., Aldridge, V. J., McCallum, W. C., \& Winter, A. L. (1964). Contingent negative variation: an electric sign of sensorimotor association and expectancy in the human brain. Nature, 203, 380-384.

Weerts, T. C., \& Lang, P. J. (1973). The effects of eye fixation and stimulus and response location on the contingent negative variation (CNV). Biological Psychology, 1, 1-19.

Wilkinson, R. T., \& Ashby, S. M. (1974). Selective attention, contingent negative variation and the evoked potential. Biological Psychology, 1, 167-179.

Wilkinson, R. T., \& Spence, M. V. (1973). Determinants of the post-stimulus resolution of contingent negative variation (CNV). Electroencephalography and Clinical Neurophysiology, 35, 503-509. 


\section{Figure legends}

Figure 1. Means from Simson et al. (1977b), Roberts et al. (1994) and Verleger et al. (2006)

for comparison (measured with a ruler from their figures). Absolute values have been used to match Simson et al.'s original figure. Note that sites Fz-Pz are approximations of site locations in Simson et al., and that Roberts et al. and Verleger et al. did not use $\mathrm{FCz}$ and $\mathrm{CPz}$ electrode sites.

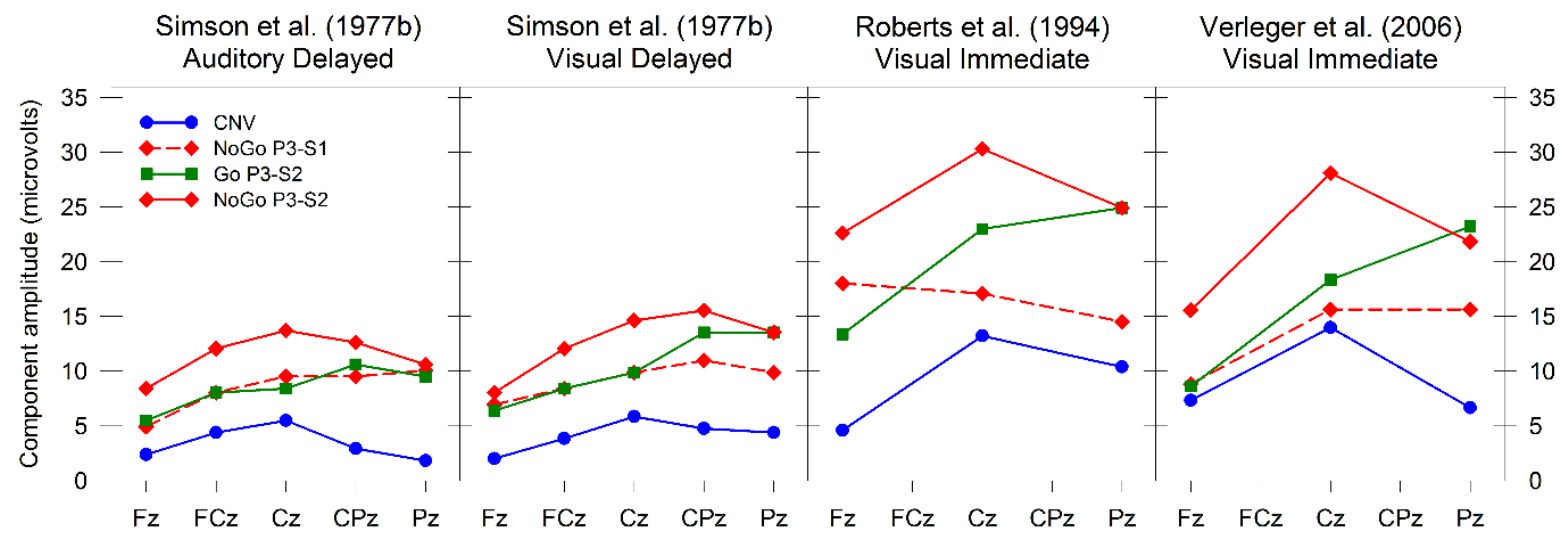


Figure 2. Grand mean ERP waveforms across conditions to cues prior to Go (green) and NoGo (red) targets. A long pre-cue interval is used for similarity with Simson et al. (1977b). Cue onset occurs at $-1000 \mathrm{~ms}$ and target onset at $0 \mathrm{~ms}$. Little difference is observed between Go and NoGo stimulus types, since the cue was uninformative as to target identity.

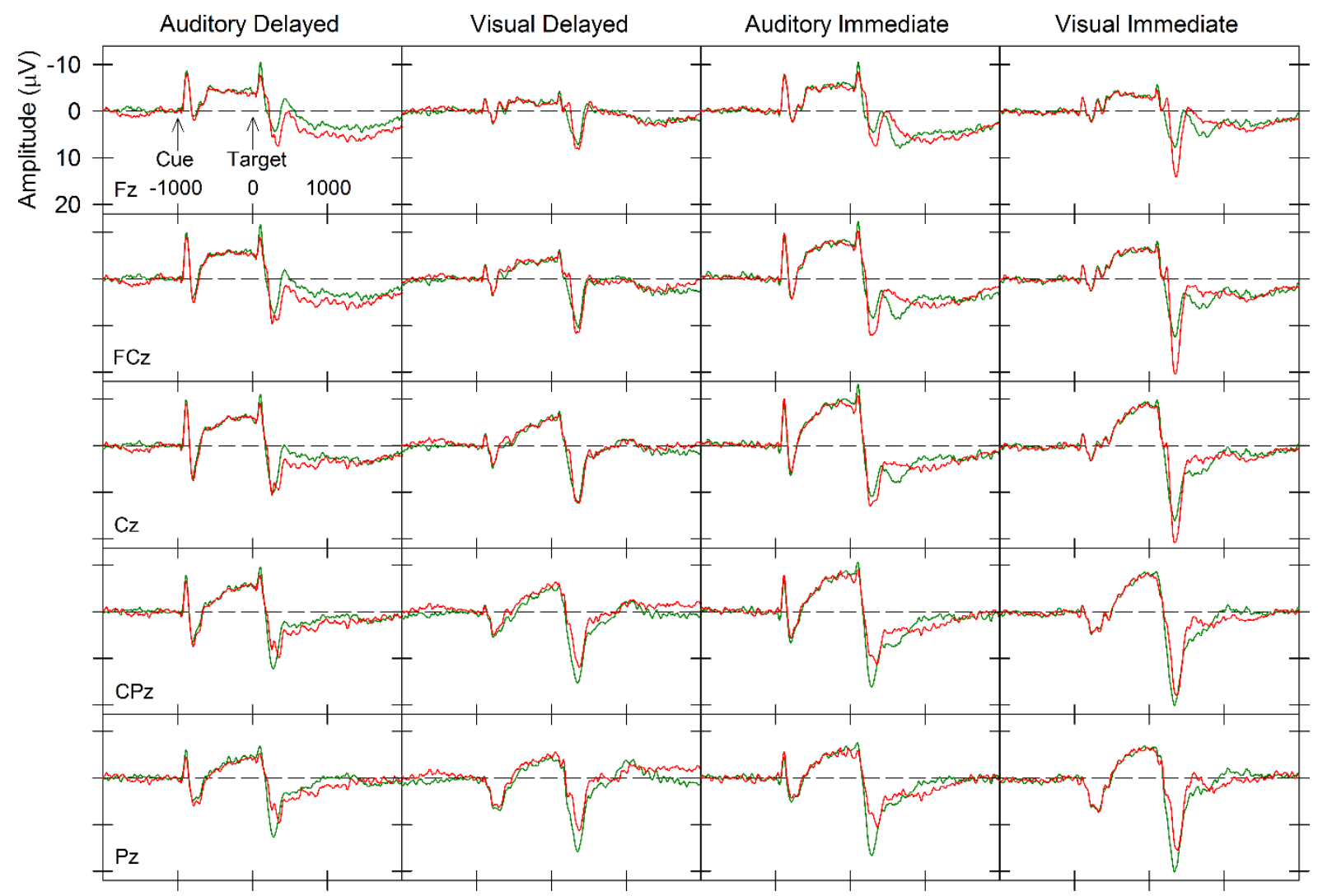


Figure 3: Means and standard errors from this study. The distinctive NoGo P3 anteriorisation relative to Go P3 is retained after CNV subtraction.

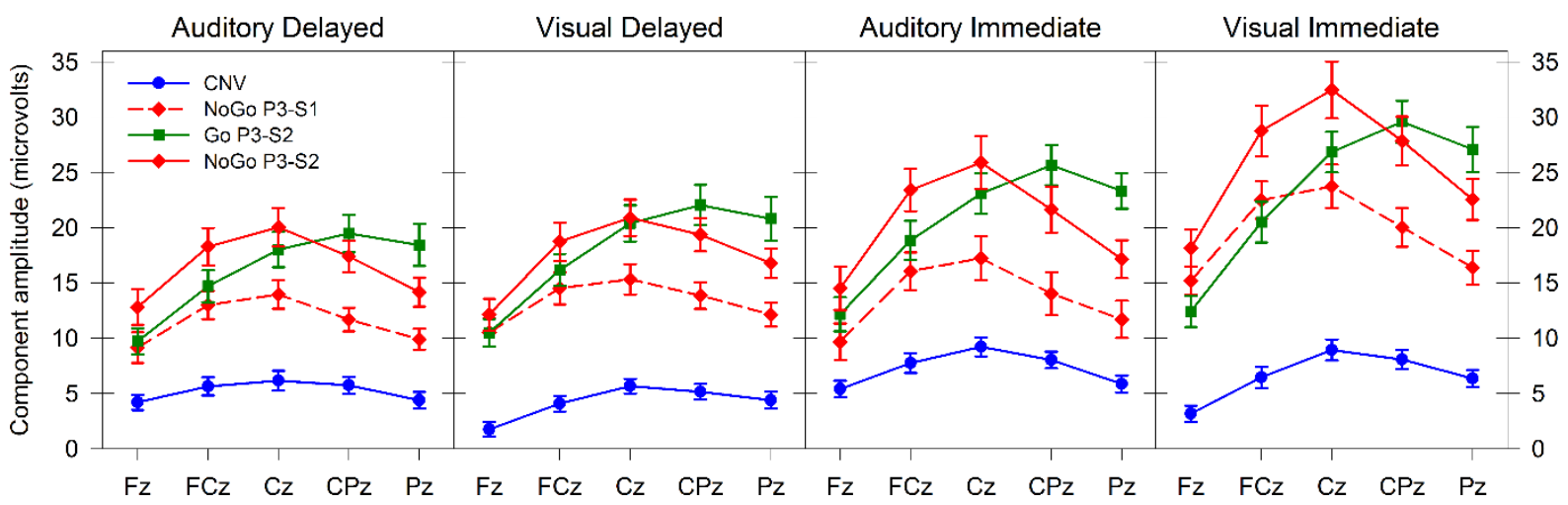


Figure 4: Topographic headmaps. For the CNV, darkest blue represents -11.5 microvolts while darkest red represents +4.5 microvolts. For the P3 (all on same scale), darkest blue represents +1 microvolts, while darkest red represents +33 microvolts.

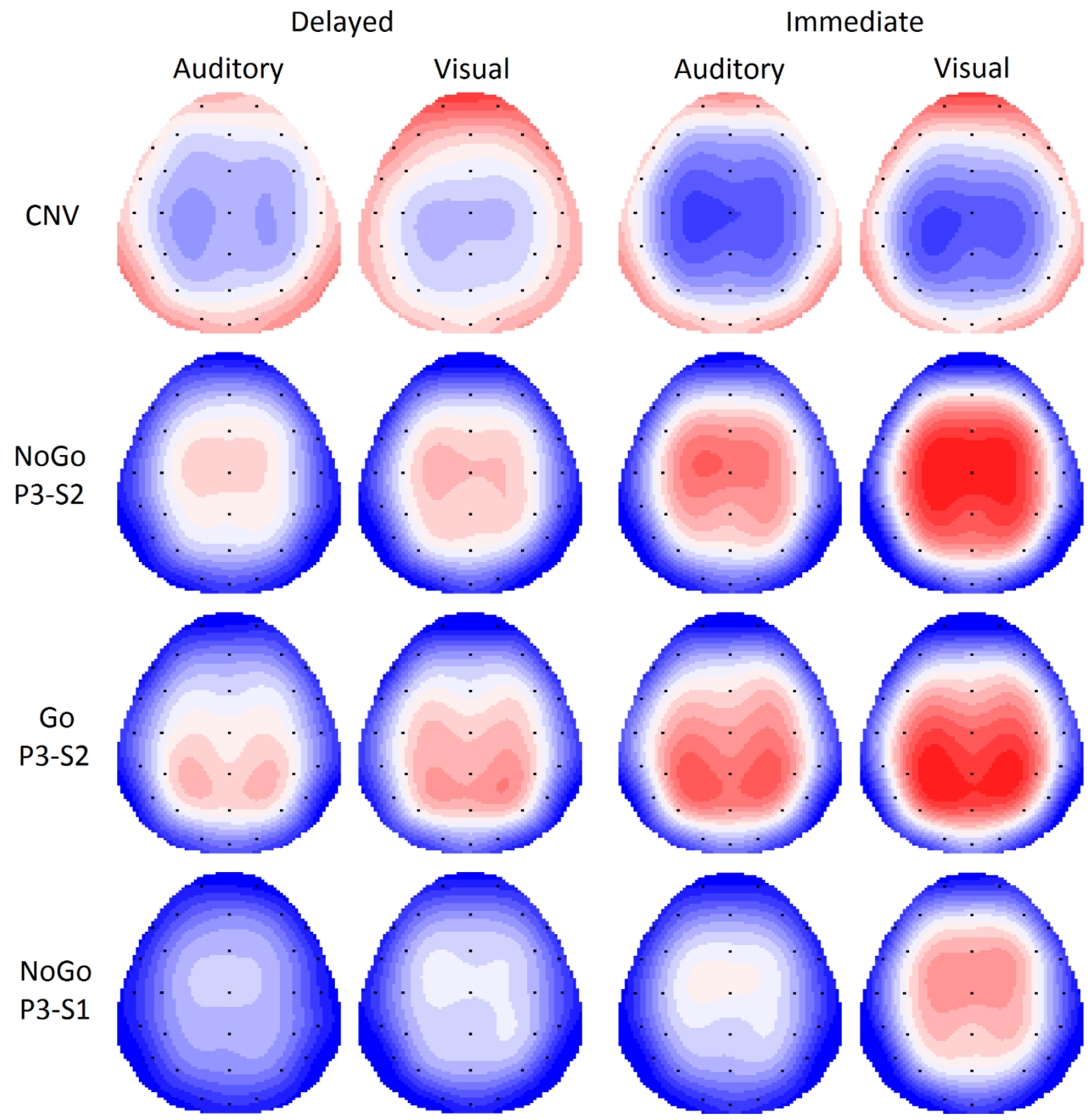


Figure 5. Grand mean ERP waveforms to Go (green) and NoGo (red) targets.

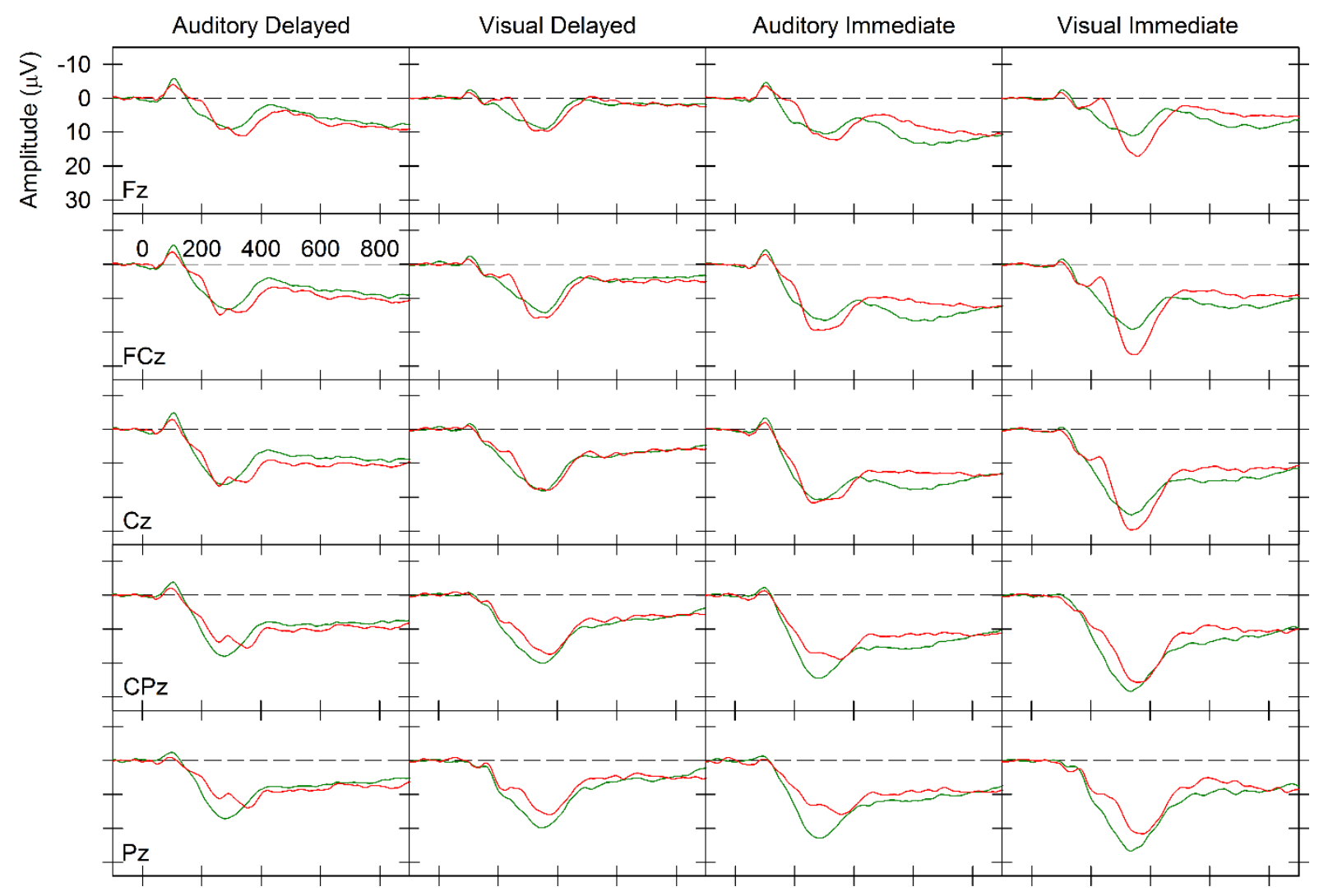

